\title{
Evaluación de la Actividad Antioxidante de Cnidoscolus chayamansa (Chaya), Euphorbia prostrata (Hierba de la Golondrina) y Jatropha dioica (Sangre de Drago) en Ratas Wistar Inducidas a Hiperglicemia
}

\author{
Evaluation of the Antioxidant Activity of Cnidoscolus chayamansa (Chaya), \\ Euphorbia prostrata (Herb of the Swallow) and Jatropha dioica (Drago blood) \\ in Wistar rats Induced to Hyperglycemia
}

\author{
Ramón Valenzuela Soto'; Joel Jiménez Villarreal²; Rubén García Garza³ Nadia D. Betancourt Martínez; \\ Rodrigo Lozoya Martínez ; Daniel Almaráz Celis ${ }^{5}$ \& Javier Morán Martínez ${ }^{4,5}$
}

\begin{abstract}
VALENZUELA, S. R.; JIMÉNEZ, V. J.; GARCÍA, G. R.; BETANCOURT, M. N. D.; LOZOYA, M. R.; ALMARÁZ, C. D. \& MORÁN, M. J. Evaluación de la actividad antioxidante de Cnidoscolus chayamansa (chaya), Euphorbia prostrata (hierba de la golondrina) y Jatropha dioica (sangre de drago) en ratas Wistar inducidas a hiperglicemia. Int. J. Morphol., 37(1):36-42, 2019.
\end{abstract}

RESUMEN: Estudios recientes han demostrado que los compuestos activos presentes en extractos de C. chayamansa, E. prostrata y $J$. dioica tienen propiedades antioxidantes. Los resultados obtenidos en nuestro estudio fueron compuestos fenólicos solubles mostraron en $C$. chayamansa $6,34, E$. prostrata $10,67, J$. dioica $1,83 \mathrm{mg}$ equiv de ácido gálico/gm BS respectivamente. Los antioxidantes solubles en agua por el método ABTS fueron para C. chayamansa 5.9, E. prostrata 12.7 y para J. dioica $2.5 \mathrm{mM}$ equiv. de trolox/gr BS. Los resultados histopatológicos muestran una mejoría en los tejidos tratados con los extractos después de la inducción a hiperglicemia.

PALABRAS CLAVE; Hiperglicemia; Ratas Wistar; Extractos.

\section{INTRODUCCIÓN}

La diabetes mellitus es una enfermedad crónicodegenerativa que manifiesta un trastorno metabólico que cursa con hiperglucemia, debido a la deficiencia en la secreción de insulina (Instituto Nacional de Geografía e Informática, 2002; Ricciardi, 2005). Las complicaciones crónicas de tipo microvascular como la retinopatía, nefropatía y neuropatía periférica afectan nervios de pies y manos, y las complicaciones macrovasculares como la enfermedad cerebro-vascular y la cardiopatía isquémica, esta última considerada la primera causa de mortalidad son consecuencias de la diabetes mellitus (Barquera et al., 2003; Dorantes \& Martínez, 2004). En el año 2005 el umbral de la glicemia en ayunas bajó de 6,1 a $5,6 \mathrm{mmol} / \mathrm{L}$, de acuerdo con los criterios de la American Diabetes Association (ADA) para la intolerancia a la glucosa (Liberopoulos et al., 2005).
Por otro lado, el potencial de la medicina herbolaria presenta nuevas alternativas que ayudan a incrementar la calidad de vida de los pacientes diabéticos y reduciendo los efectos secundarios de los medicamentos. En México se conocen alrededor de 150 plantas que han sido tradicionalmente utilizadas para el tratamiento de la diabetes, incluyendo a la $C$. chayamansa (chaya), E. prostrata (hierba de la golondrina) y J. dioica (sangre de drago), las cuales pertenecen a la familia Euphorbiaceae (Rooss-Ibarra \& Molina-Cruz, 2002; Quezada et al., 2007).

Diversas investigaciones han reportado algunos compuestos químicos que se han identificado en estas plantas son; glucósidos cianogénicos (Molina et al., 1999), compuestos fenólicos, terpenoides como la citlalitriona,

\footnotetext{
${ }^{1}$ Universidad Politécnica de Gómez Palacio, Gómez Palacio, Durango, México.

${ }^{2}$ Departamento de Investigación, Escuela de Medicina Unidad Norte, Universidad Autónoma de Coahuila, Piedras Negras, Coahuila, México.

${ }^{3}$ Departamento de Histología, Facultad de Medicina, Universidad Autónoma de Coahuila. Unidad Torreón. Torreón, Coahuila, México.

${ }^{4}$ Departamento de Biología Celular y Ultraestructura, Centro de Investigación Biomédica, Facultad de Medicina, Universidad Autónoma de Coahuila.Unidad Torreón. Torreón, Coahuila, México.

${ }^{5}$ Coordinación de Investigación, Escuela de Medicina, Universidad Autónoma de Durango. Gómez Palacio, Durango, México.
} 
VALENZUELA, S. R.; JIMÉNEZ, V. J.; GARCÍA, G. R.; BETANCOURT, M. N. D.; LOZOYA, M. R.; ALMARÁZ, C. D. \& MORÁN, M. J. Evaluación de la actividad antioxidante de Cnidoscolus chayamansa (chaya), Euphorbia prostrata (hierba de la golondrina) y Jatropha dioica (sangre de drago) en ratas Wistar inducidas a hiperglicemia. Int. J. Morphol., 37(1):36-42, 2019.

jatrofona, riolosatriona, R-sitosterol y taninos (Chen et al., 1994; Biblioteca Digital de la Medicina Tradicional Mexicana, 2009). Los antioxidantes pueden inhibir o retardar el proceso oxidativo, interfiriendo con la iniciación o propagación de las reacciones en cadena de la auto oxidación; estos elementos también tienen la función de eliminar de nuestro organismo los radicales libres. El exceso de radicales libres está relacionado con una mayor incidencia de diversas enfermedades degenerativas (Patricò \& Delanty, 2000). Como cáncer, enfermedades cardiacas, inflamación, artritis, disfunción cerebral, aceleración del envejecimiento (Finkel \& Holbrook, 2000). Estudios recientes han demostrado que algunos fitoquímicos presentes en los extractos de las hojas de C. chayamansa (chaya), E. prostrata (hierba de la golondrina) y $J$. dioica (sangre de drago) tienen propiedades antioxidantes. Por lo tanto el objetivo de este estudio fue detectar y evaluar compuestos con capacidad antioxidante en extractos acuosos en estas tres plantas en un modelo in vivo.

\section{MATERIAL Y MÉTODO}

Material Biológico. Fueron usadas las hojas de $C$. chayamansa (Chaya), E. prostrata (Hierba de la Golondrina), J. dioica (Sangre Drago) y recolectadas en la Región Lagunera de Durango (Gómez Palacio y Lerdo Durango) zona norte de México, parte del material que se depositó en el herbario de la Facultad de Ciencias Biológicas de la Universidad Autónoma de Nuevo León, para su identificación, archivo y obtención del número de voucher.

Preparación del material vegetal y extracto. Las hojas se secaron a temperatura ambiente por 10 días y fueron molidas con una licuadora casera hasta obtener un polvo fino (Navarro García et al., 2006). Se disolvieron $60 \mathrm{~g}$ de muestra seca en $300 \mathrm{~mL}$ de agua destilada estéril a $90{ }^{\circ} \mathrm{C}$ por $1 \mathrm{~h}$ con agitación continua y después de filtro y se almacenaron en refrigeración a $5^{\circ} \mathrm{C}$ hasta su análisis.

Cuantificación del contenido de fenólicos totales. El contenido fenólico total se determinó usando una modificación del método Folin-Ciocalteau (Singleton \& Rossi, 1965) publicado por Esparza Rivera et al. (2006). Se diluyó $30 \mathrm{~mL}$ de muestra en $270 \mathrm{~mL}$ de agua destilada después se le agrego $1,5 \mathrm{~mL}$ de reactivo Folin-Ciocalteau (Sigma-Aldrich, St. Louis MO, EU) diluido (1:15), agitando durante $10 \mathrm{~s}$. Después de $5 \mathrm{~min}$ se añadió $1,2 \mathrm{~mL}$ de solución de carbonato de sodio $(7,5 \%$ p/v) agitándose 10 s. La solución se colocó a baño maría a $45^{\circ} \mathrm{C}$ por 15 min, y luego se dejó enfriar a temperatura ambiente. La absorbancia de la solución fue leída a $765 \mathrm{~nm}$ en un espectrofotómetro HACH UDR 4000. El contenido fenólico se calculó mediante una curva patrón usando ácido gálico (Sigma, St. Louis, Missouri, EU) como estándar, y los resultados se reportaron en mg de ácido gálico equivalente por g demuestra base seca (mg equiv AG.g-1 BS), los análisis se realizaron por triplicado.

Capacidad antioxidante equivalente en Trolox por el método DPPH+ y ABTS+. La capacidad antioxidante fue determinada por los métodos de DPPH+ y ABTS+, por triplicado, como sigue; se utilizó el método in vitro DPPH+ modificado por Brand-Williams et al. (1995). Se preparó una solución de DPPH ${ }^{*}$ (Aldrich, St. Louis, MO, EU) en metanol, ajustando la absorbancia de la solución a 1,100 \pm 0,010 a una longitud de onda de $515 \mathrm{~nm}$. Para la determinación de capacidad antioxidante se mezclaron $50 \mathrm{~mL}$ de muestra y $950 \mathrm{~mL}$ de solución DPPH+, y después de 3 min de reacción se analizó la absorbancia de solución a $515 \mathrm{~nm}$ y se realizó una curva estándar con Trolox (Aldrich, St. Louis, MO, EU). Para el Método in vitro de ABTS+ se utilizó el método de Esparza Rivera et al. Se preparó una solución de $\mathrm{ABTS}^{\circ}+$ con $40 \mathrm{mg}$ de ABTS (Aldrich, St. Louis, Missouri, EU) y 1,5 g de dióxido de manganeso (Fermont, Nuevo León, México) en $15 \mathrm{~mL}$ de agua destilada. La mezcla fue agitada vigorosamente y se dejó reposar durante 20 min. Después, la solución se filtró en papel Whatman 40 (GE Healthcare UK Limited, Little Chalfont, Buckinghamshire, Reino Unido), y la absorbancia se ajustó a $0,700 \pm 0,010$ con una longitud de onda de $734 \mathrm{~nm}$, usando una solución buffer de fosfato $5 \mathrm{mM}$. Para la determinación de capacidad antioxidante se mezclaron $100 \mathrm{~mL}$ de muestra y $1 \mathrm{~mL}$ de solución $\mathrm{ABTS}^{*}+$, y después de 60 y 90 s de reacción se analizó la absorbancia de la muestra a $734 \mathrm{~nm}$. Se realizó una curva estándar con Trolox (Aldrich, St. Louis, MO, EU), y los resultados se reportaron como capacidad antioxidante equivalente en $\mathrm{mM}$ equivalente en Trolox por $\mathrm{g}$ base fresca (mM equiv Trolox.gm-1 BF), todas las muestras se realizaron por triplicado.

\section{Tejidos de hígado y riñón de Ratas Wistar.}

Animales. Se utilizaron 30 ratas Wistar macho con un rango de peso de 250 a $300 \mathrm{~g}$, y se mantuvieron en el bioterio de la unidad de fitoquímica de la Facultad de Medicina de la Universidad Autónoma de Coahuila, bajo condiciones controladas de temperatura $25{ }^{\circ} \mathrm{C}$, humedad $60 \%$ y ciclos de luz/oscuridad (12 h), alimentadas ad libitum.

Grupos experimentales. Se emplearon 5 ratas Wistar macho para cada grupo como sigue; control, ratas diabéticas, diabéticas tratadas con glibenclamida, $C$. chayamansa, $E$. prostrata, J. dioica. Posteriormente se administró una dosis única de $40 \mathrm{mg} / \mathrm{Kg}$ de peso corporal (PC) de 
estreptozotocina (SZT) en buffer de citrato al $0,1 \mathrm{M}(\mathrm{pH}$ 4,5) por vía intraperitoneal, dos horas después de la inyección se presentó una hiperglicemia en sangre, y seis horas después de la inducción se presentó una hipoglicemia en sangre. Finalmente se desarrolló la hiperglicemia en sangre debido a las anormalidades que se generan en la función de las células b originando diabetes tipo 2 caracterizada por concentración de glucosa de $180 \mathrm{mg} / \mathrm{dL}$ y ligero daño a las células pancreáticas, se mantuvieron diabéticas por 7 días y después se inicio el tratamiento por 30 días con cada extracto por medio de una sonda intra gástrica.

Análisis histopatológico. Para evaluar el efecto de los extractos a nivel del tejido, los animales fueron sacrificados por dislocación cervical al final del tratamiento y fueron recolectados los órganos de hígado y riñón y colocados en formalinaneutra al $10 \%$. Los órganos fueron procesados mediante la técnica histológica convencional y fueron embebidos en bloque de parafina, los cortes de los tejidos de realizaron a $5 \mu \mathrm{m}$ con un micrótomo y fueron teñidos con hematoxilina y eosina y después de la tinción, fueron montados en resina sintética con un cubreobjetos en se realizó el análisis histológico en un microscopio de campo claro.

Análisis estadístico. Los resultados obtenidos fueron analizados usando ANOVA con significancia de 0,05 . con una prueba de comparación múltiple de Tukey $\mathrm{P}<0,05$ usando el paquete estadístico SPSS versión 20.

\section{RESULTADOS}

Material biológico. Las plantas fueron identificadas y se les asignaron los voucher siguientes: 22388 para las hojas de $C$. chayamansa, 07807 para E. prostrata y 34077 para J. dioica.

Cuantificación de fenólicos totales. En la Tabla I se muestra la presencia de compuestos fenólicos y antioxidantes en los extractos acuosos de C. chayamansa, E. prostrata y $J$. dioica se encontró una mayor concentración de estos compuestos en E. prostrata. En la Tabla II se muestra que la sangre de drago presenta mayor cantidad de flavonoides y antioxidantes en comparación a los otros dos extractos, sin embargo en este cuadro se puede apreciar que existen compuestos similares en los tres extractos.

\section{Determinación e identificación de compuestos}

En las Figuras 1, 2 y 3 se muestra la presencia de diferentes compuestos antioxidantes y flavonoides en extractos acuosos de C. chayamansa, E. prostrata, J. dioica.

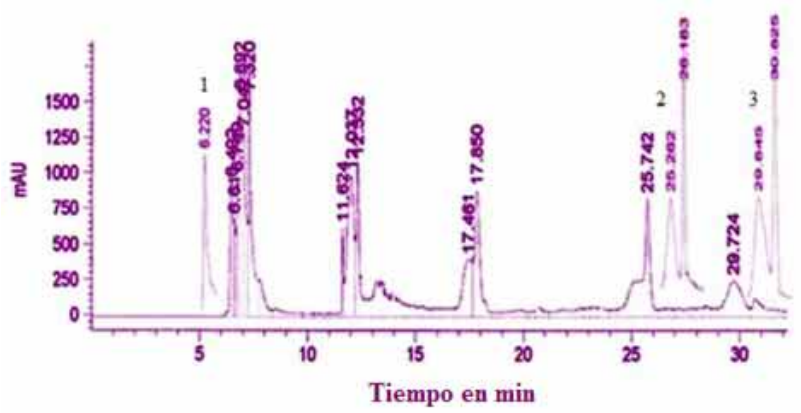

Fig. 1. Presencia de compuestos antioxidantes y flavonoides en $C$. chayamansa; 1) Ácido p-hidroxibenzoico, 2) Catequina, 3) Epicatequina.

Tabla I. Cuantificación de la actividad antioxidante y contenido fenólico.

\begin{tabular}{lccc}
\hline & $\begin{array}{c}\text { Fenólicos mg equivalente de } \\
\text { Extractos (acuoso) }\end{array}$ & $\begin{array}{c}\text { (ABTS) mMol equivalente de } \\
\text { Trolox/g base seca }\end{array}$ & $\begin{array}{c}\text { (DPPH) mMol equival ente de } \\
\text { Trolox/g base seca }\end{array}$ \\
\hline C. chayamansa & $6,34^{*}$ & $5,9^{*}$ & $1,1^{*}$ \\
E. prostrata & 10,67 & 12,7 & 9,53 \\
J. dioica & 1,83 & 2,5 & 1,03 \\
\hline
\end{tabular}

* Resultado publicado por Valenzuela Soto et al. (2015).

Tabla II. Presencia de compuestos fenolicos y flavonoides de las infusiones.

\begin{tabular}{lccc}
\hline Compuestos fenolicos y Flavonoides & \multicolumn{3}{c}{ Extractos Acuosos } \\
& C. chayamansa & E. prostrata & $J$. dioica \\
\hline Ácido Clorogénico & - & + & + \\
Ácido Gálico & - & - & + \\
Ácido Siríngico & - & + & + \\
Catequina & + & + & + \\
Epi Catequina & + & + & + \\
Rutina & - & + & + \\
Ácido $p$-Hidroxibenzoico & + & + & + \\
\hline
\end{tabular}


VALENZUELA, S. R.; JIMÉNEZ, V. J.; GARCÍA, G. R.; BETANCOURT, M. N. D.; LOZOYA, M. R.; ALMARÁZ, C. D. \& MORÁN, M. J. Evaluación de la actividad antioxidante de Cnidoscolus chayamansa (chaya), Euphorbia prostrata (hierba de la golondrina) y Jatropha dioica (sangre de drago) en ratas Wistar inducidas a hiperglicemia. Int. J. Morphol., 37(1):36-42, 2019.

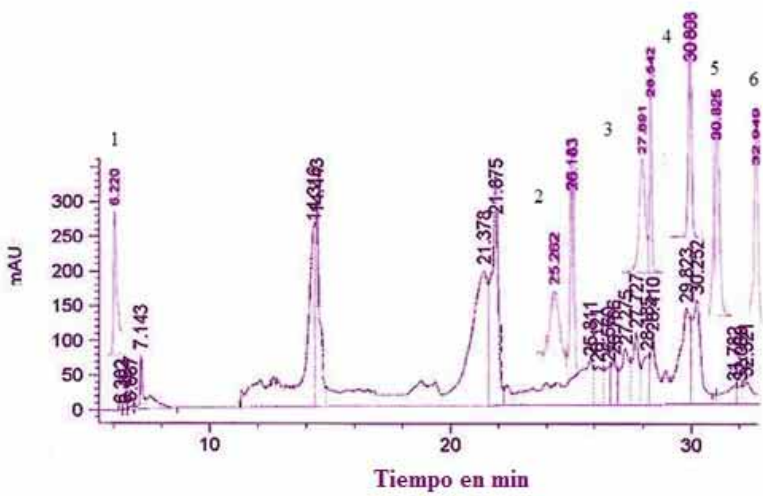

Fig. 2. Presencia de compuestos antioxidantes y flavonoides en $E$. prostrata; 1) Ácido p-hidroxibenzoico, 2) Catequina, 3) Ácido clorogénico, 4) Ácido siríngico, 5) Epicatequina, 6) Rutina.

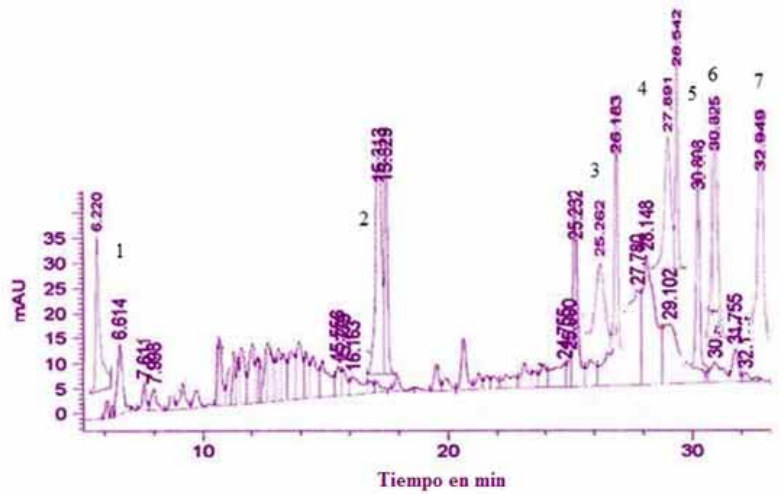

Fig. 3. Presencia de compuestos antioxidantes y flavonoides en $J$. dioica; 1) Ácido p-hidroxibenzoico, 2) Ácido gálico, 3) Catequina, 4) Ácido clorogénico, 5) Ácido siríngico, 6) Epicatequina, 7) Rutina.
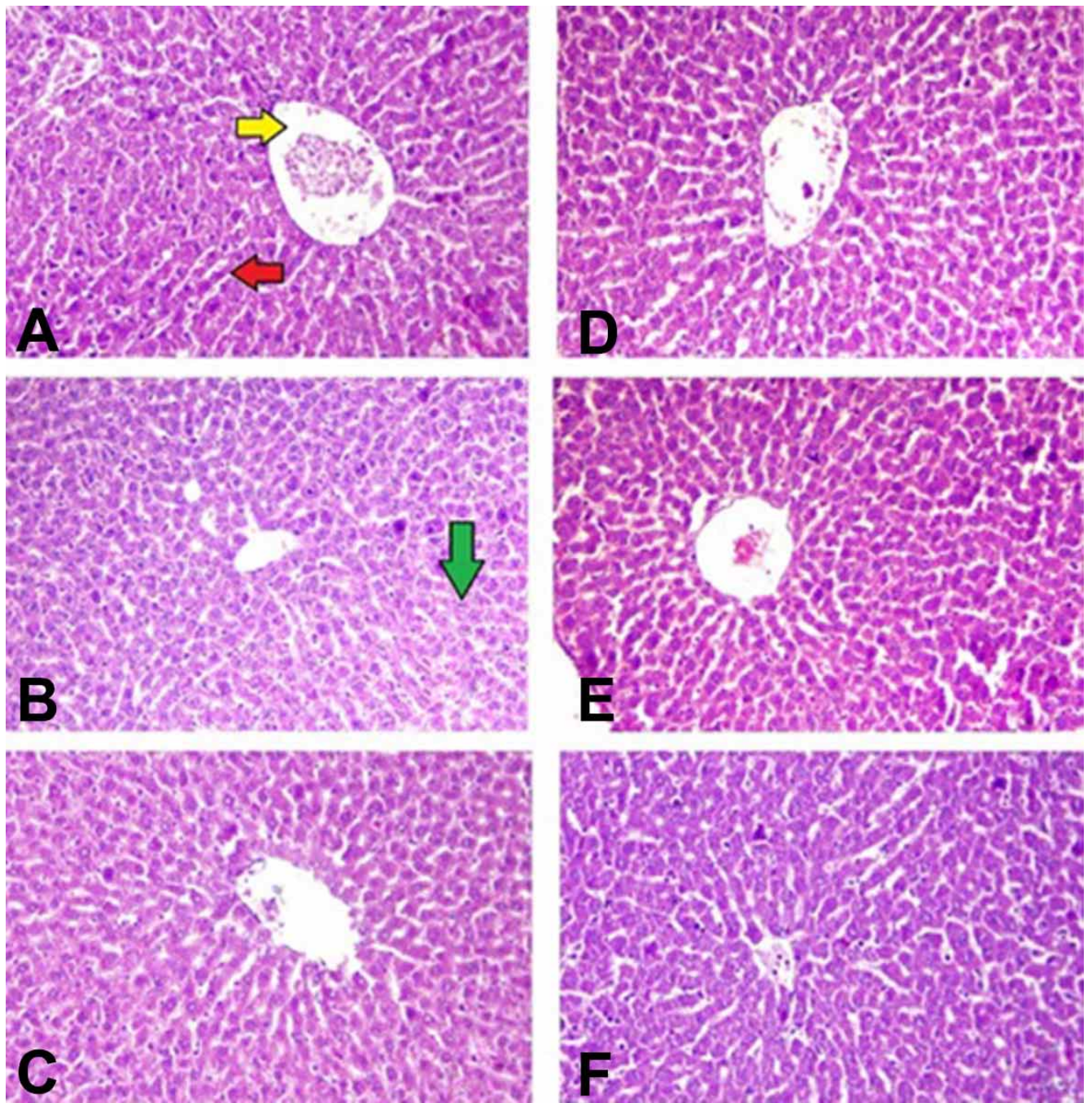

Fig. 4. Análisis histopatológico de hígado de los grupos experimentales; control, ratas diabéticas, diabéticas tratadas con glibenclamida, C. chayamansa, E. prostrata, J. dioica.
R e s u l t a d o $s$ histopatológicos. Microfotografías de hígado de grupo control (A) y experimentales (B-F). En las ratas del grupo control (A) se observaron hepatocitos (flecha roja) y venas centrales de características histológicas normales. En el grupo de ratas con diabetes (B) se observó la presencia de abundantes vacuolas en el citoplasma de los hepatocitos. En los grupos de diabetes más glibenclamida (C) $C$. chayamansa (D), J. dioica (E) y E. prostrata (F) se encontraron los mismos hallazgos observados en el grupo control. (Embebido en parafina. HyE. 10X) (Fig. 4). 
VALENZUELA, S. R.; JIMÉNEZ, V. J.; GARCÍA, G. R.; BETANCOURT, M. N. D.; LOZOYA, M. R.; ALMARÁZ, C. D. \& MORÁN, M. J. Evaluación de la actividad antioxidante de Cnidoscolus chayamansa (chaya), Euphorbia prostrata (hierba de la golondrina) y Jatropha dioica (sangre de drago) en ratas Wistar inducidas a hiperglicemia. Int. J. Morphol., 37(1):36-42, 2019.

Microfotografías de riñón de grupo control (A) y experimentales (B-F). En las ratas del grupo control (A) se observaron glomérulos (flecha blanca) y túbulos contorneados (flecha amarilla) de características histológicas normales.

En el grupo de ratas con diabetes (B) se observó la presencia de glomérulos congestionados y retraídos (fle- cha blanca). En el grupo de diabetes más glibenclamida (C) no se observaron alteraciones. En el grupo tratado con C. chayamansa (D) se observó vacuolización del citoplasma de los túbulos contorneados (flecha amarilla), con áreas de destrucción de su citoplasma. En los grupos tratados con, J. dioica (E) y E. prostrata (F) se encontraron los mismos hallazgos observados en el grupo control. (Embebido en parafina. HyE. 10X) (Fig. 5).
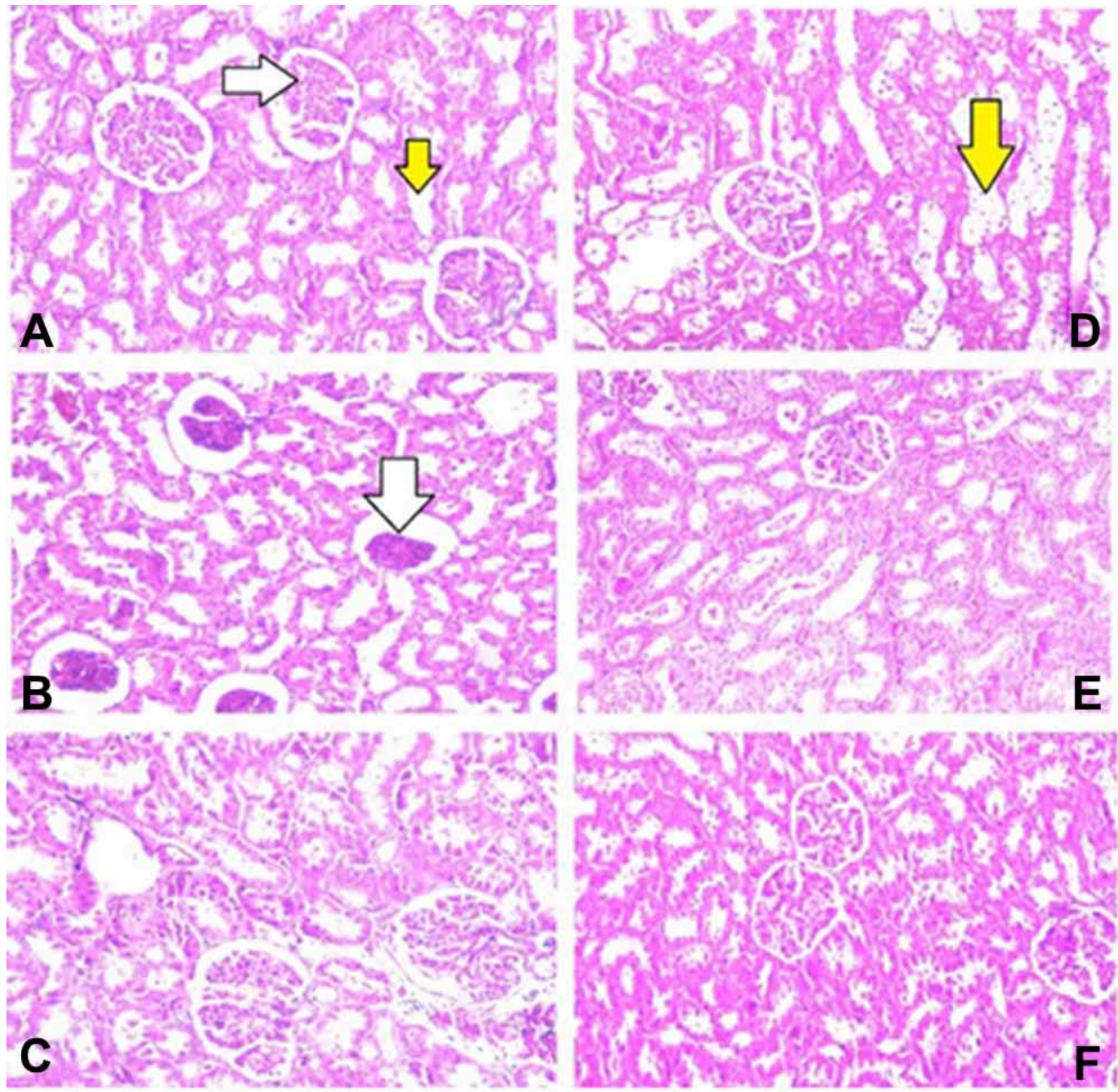

Fig. 5. Análisis histopatológico de riñón de los grupos experimentales; control, ratas diabéticas, diabéticas tratadas con glibenclamida, C. chayamansa, E. prostrata, J. dioica.

\section{DISCUSIÓN}

Recientes investigaciónes han demostrado algunos compuestos fitoquímicos presentes en los extractos de las hojas de C. chayamansa, E. prostrata y J. dioica tienen propiedades antioxidantes. Por lo que es necesario realizar más estudios para comprobar dichas propiedades en estas plantas. Fue- ron detectados los siguientes compuestos fenólicos y flavonoides en los extractos evaluados: rutina catequina, epicatequina, ácido clorogénico, siringico y gálico, phidroxibenzoico, capsaicina. Lima et al. (2009) menciona la posible presencia de catequina y epicatequina en infusión de 
Camellia sinensis $L$. Estos hallazgos se corroboran en nuestro estudio, el contenido fenólico obtenido en el presente estudio fue $10,67 \mathrm{mg}$ equiv. de ác. gálico/mL en la infusión de $E$. prostrata y $1,83 \mathrm{mg}$ equiv de ácido gálico/mL en la infusión de tallo de J. dioica en comparación con los resultados encontrados por Gutiérrez Zavala et al. (2007), Loarca-Piña et al. (2010) y Mercado-Mercado et al. (2013). Para comparar el efecto antioxidante existen pocos estudios que muestren resultados histopatológicos con el uso de estas tres plantas, sin embargo, en un estudio realizado por Kuti \& Torres (1996), se evaluó el efecto del té de chaya ( $10 \mathrm{~g}$ de hojas por litro de agua) administrado ad libitum a conejos sanos y diabéticos inducidos con estreptozotocina, en este experimento se observó que el nivel de glucosa en ayuno disminuyó gradualmente después de 6 horas de iniciado el experimento. Sin embargo, la dosis eficaz y el mecanismo de actividad hipoglucemiante no fueron evaluados. En otro estudio realizado por AlarconAguilara et al. (1998), utilizó la Cnidoscolus chayamansa (Chaya) para determinar su efecto antihiperglicémico, los resultados no confirmaron que dichos extractos manejados en el estudio tuvieran actividad hipoglicemiante. Por otro lado, Aguilera-Carbo et al. (2008) cuantificaron uno de los compuestos fenólicos y terpenoides presentes en J. dioica, por lo que se podría considerar como una fuente alternativa de propiedades relacionadas a la salud, como acciones antiesteroescleróticas, propiedades anticarcinogénicas resultando en una posible reducción de cáncer de colon humano, próstata, cervical, lengua, esófago y piel, además de ser utilizado en la industria alimentaria como posible agente antioxidante. Nuestros resultados muestran una reducción del daño en los grupos tratados con los diferentes extractos en comparación con las ratas inducidas a diabetes, sin embargo, faltas más estudios y pruebas para elucidar el posible mecanismo de acción y cuáles de los antioxidantes encontrados en los extractos son los que están ejerciendo su fuerza antioxidante ante el daño.

\section{CONCLUSIÓN}

Los extractos de las plantas probadas, muestran un efecto antioxidante en comparación con los grupos inducidos a diabetes. Los resultados de los métodos de Folin-Ciocalteau, ABTS y DPPH, muestran que el extracto de la E. prostrata, contiene la mayor concentración de fenólicos totales y/o de compuestos. Los resultados de los análisis por HPLC muestran que la J. dioica. contiene mayor presencia de flavonoides y de compuestos antioxidantes, pero esto no indica que sea la más eficaz contra la diabetes. Los extractos a base de $C$. chayamansay $E$. prostrata mostraron una mayor capacidad antioxidantes y flavonoides presentes en los tres extractos (catequina, epi catequina y ácido p-hidroxibenzoico) a esta capacidad se le puede atribuir el efecto hipoglucemiante.
VALENZUELA, S. R.; JIMÉNEZ, V. J.; GARCÍA, G. R.; BETANCOURT, M. N. D.; LOZOYA, M. R.; ALMARÁZ, C. D. \& MORÁN, M. J. Evaluation of the antioxidant activity of Cnidoscolus chayamansa (chaya), Euphorbia prostrata (herb of the swallow) and jatropha dioica (drago blood) in wistar rats induced to hyperglycemia. Int. J. Morphol., 37(1):36-42, 2019.

SUMMARY: Recent studies have shown that the active compounds present in extracts of C. chayamansa, E. prostrata and $J$. dioica have antioxidant properties. The results obtained in our study were soluble phenolic compounds showed in C. chayamansa 6.34 , E. prostrata 10.67 , J. dioica $1.83 \mathrm{mg}$ equiv of gallic acid/gm $\mathrm{BS}$ respectively. The antioxidants soluble in water by the ABTS method were for C. chayamansa 5.9, E. prostrata 12.7 and for $J$. dioica $2.5 \mathrm{mM}$ equiv. of trolox/gr BS. The histopathological results show an improvement in the tissues treated with the extracts after the induction to hyperglycemia.

KEY WORDS: Hyperglycemia; Wistar rats; Extracts.

\section{REFERENCIAS BIBLIOGRÁFICAS}

Aguilera-Carbo, A. F.; Augur, C.; Prado-Barragan, L. A.; Aguilar, C. N. \& Favela-Torres, E. Extraction and analysis of ellagic acid from novel complex sources. Chem. Pap., 62(4):440-444, 2008.

Alarcon-Aguilara, F. J.; Roman-Ramos, R.; Perez-Gutierrez, S.; AguilarContreras, A.; Contreras-Weber, C. C. \& Flores-Saenz, J. L. Study of the anti-hyperglycemic effect of plants used as antidiabetics. $J$. Ethnopharmacol., 61(2):101-10, 1998.

Barquera, S.; Tovar-Guzmán, V.; Campos-Nonato, I.; Gónzález-Villalpando, C. \& Rivera-Dommarco, J. Geography of diabetes mellitus mortality in Mexico: an epidemiologic transition analysis. Arch. Med. Res., 34(5):407-14, 2003.

Biblioteca Digital de la Medicina Tradicional Mexicana (BDMTM). Atlas de las Plantas de la Medicina Tradicional Mexicana. Ciudad de México, Biblioteca Digital de la Medicina Tradicional Mexicana, 2009. Disponible en: http://www.medicinatradicionalmexicana.unam.mx/ monografia.php?1=3\&t=\&id=7483.

Brand-Williams, W.; Cuvelier, M. E. \& Berset, C. Use of a free radical method to evaluate antioxidant activity. L. W. T. Food Sci. Technol., 28(1):25-30, 1995.

Chen, Z. P.; Cai, Y. \& Phillipson, J. D. Studies on the anti-tumour, antibacterial, and wound-healing properties of dragon's blood. Planta Med., 60(6):541-5, 1994.

Dorantes, C. A. \& Martínez, S. C. Endocrinología Clínica. 2a ed. Ciudad de México, Manual Moderno, 2004.

Esparza Rivera, J. R.; Stone, M. B.; Stushnoff, C.; Pilon-Smits, E. \& Kendall, P. A. Effects of ascorbic acid applied by two hydrocooling methods on physical and chemical properties of green leaf lettuce stored at $5^{\circ} \mathrm{C}$. $J$. Food Sci., 71(3):S270-6, 2006.

Finkel, T. \& Holbrook, N. J. Oxidants, oxidative stress and the biology of ageing. Nature, 408(6809):239-47, 2000.

Gutiérrez Zavala, A.; Ledesma Rivero, L.; García García, I. \& Grajales Castillejos, O. Capacidad antioxidante total en alimentos convencionales y regionales de Chiapas, México. Rev. Cub. Salud Pública, 33(1):1-7, 2007.

Instituto Nacional de Geografía e Informática (INEGI). Mortalidad General por Edad y Causas Detalladas. Ciudad de México, Sistemas Nacionales Estadístico y de Información Geográfica, Instituto Nacional de Geografía e Informática, 2002. 
VALENZUELA, S. R.; JIMÉNEZ, V. J.; GARCÍA, G. R.; BETANCOURT, M. N. D.; LOZOYA, M. R.; ALMARÁZ, C. D. \& MORÁN, M. J. Evaluación de la actividad antioxidante de Cnidoscolus chayamansa (chaya), Euphorbia prostrata (hierba de la golondrina) y Jatropha dioica (sangre de drago) en ratas Wistar inducidas a hiperglicemia. Int. J. Morphol., 37(1):36-42, 2019.

Kuti, J. \& Torres, E. S. Potential Nutritional and Health Benefits of Tree Spinach. In: Janick, J. (Ed.). Progress in New Crops. Arlington, ASHS Press, 1996. pp.516-20.

Liberopoulos, E. N.; Mikhailidis, D. P. \& Elisaf, M. S. Diagnosis and management of the metabolic syndrome in obesity. Obes. Rev., 6(4):28396, 2005.

Lima, J. D.; Mazzafera, P.; da Silva Moraes, W. \& da Silva, R. B. Chá: aspectos relacionados à qualidade e perspectivas. Cienc. Rural, 39(4):1258-66, 2009.

Loarca-Piña, G.; Mendoza, S.; Ramos-Gómez, M. \& Reynoso, R. Antioxidant, antimutagenic, and antidiabetic activities of edible leaves from Cnidoscolus chayamansa Mc. Vaugh. J. Food Sci., 75(2):H6872, 2010.

Mercado-Mercado, G.; de la Rosa Carrillo, L.; Wall-Medrano, A.; López Díaz, J. A. \& Álvarez-Parrilla, E. Compuestos polifenólicos y capacidad antioxidante de especias típicas consumidas en México. Nutr. Hosp., 28(1):36-46, 2013.

Molina, A.; Solórzano, M. \& Bressani, R. Procesamiento de las hojas de Chaya (Cniduscolus chayamansa; Eupharbiacea) para consumo humano: I. Cocción en agua hirviendo y almacenamiento de hojas frescas. Cienc. Acción, 6, 1999

Navarro García, V. M.; Rojas, G.; Gerardo Zepeda, L.; Aviles, M.; Fuentes, M.; Herrera, A. \& Jiménez, E. Antifungal and antibacterial activity of four selected mexican medicinal plants. Pharm. Biol., 44(4):297-300, 2006.

Patricò, D. \& Delanty, N. Oxidative injury in diseases of the central nervous system: focus on Alzheimer's disease. Am. J. Med., 109(7):577-85, 2000.

Quezada, T. T.; Martínez, V. R.; Acero, G. M. G.; López, G. M. A.; Valdivia, F. A. \& Ortíz, M. R. Evaluación químico proximal y concentración de vitamina $\mathrm{C}$ en hojas de chaya (Cnidoscolus chayamansa) con tres niveles de fertilización orgánica y química. IX Congreso de Ciencia de los Alimentos y V Foro de Ciencia y Tecnología de Alimentos, 2007.

Ricciardi, R. Sedentarism: a concept analysis. Nurs. Forum, 40(3):79-87, 2005.

Rooss-Ibarra, J. \& Molina-Cruz, A. The ethnobotany of Chaya (Cnidoscolus aconitifolius SSP. Aconitifolius breckon): A nutritious Maya Vegetable. Econ. Bot., 56:350, 2002

Singleton, V. L. \& Rossi, J. A. Colorimetry of total phenolics with phosphomolybdic-phosphotungstic acid reagents. Am. J. Enol. Vitic., 16:144-58, 1965.

Valenzuela Soto, R.; Morales Rubio, M. E.; Verde Star, M. J.; Cárdenas, A. O.; Preciado-Rangel, P.; González, J. A. \& Esparza-Rivera, J. R. Cnidoscolus chayamansa hidropónica orgánica y su capacidad hipoglucemiante, calidad nutraceutica y toxicidad. Rev. Mex. Cienc. Agríc., 6(4):815-25, 2015.

\author{
Dirección para correspondencia: \\ Javier Morán Martínez \\ Departamento de Biología Celular y Ultraestructura \\ Centro de Investigación Biomédica \\ Facultad de Medicina \\ Universidad Autónoma de Coahuila \\ Unidad Torreón \\ Torreón, Coahuila \\ México
}

Email: Javmoran@yahoo.com

Recibido : 27-07-2018

Aceptado: 17-09-2018 